\title{
Medical Education During Challenging Times
}

\author{
I.M. Karunathilake, de Abrew, A.
}

The ongoing COVID-19 pandemic has led to greater disruption to Medical Education in the South East Asian Region (SEAR) in 2021, compared to 2020. The catastrophic increase in the number of cases in countries such as India and Sri Lanka has overwhelmed national health systems. Mitigation and management of the increased disease burden have led to drastic reductions in elective procedures and routine work, with concurrent reduction of traditional learning opportunities. Depending on need and availability, in-patient wards and ambulatory care centers have been converted to COVID19 treatment centers, reflecting how management of the COVID-19 outbreak has become a national priority in many countries in the region.

Despite appearances, this does not imply a complete halt to medical education. The pandemic has provided several opportunities for medical education to adapt to the new normal. There are blessings in disguise during this challenging time (Karunathilake, 2020).

There is an increase in innovative use of technology for medical education. Many medical schools now combine face-to-face teaching with videoconferencing and online learning. Several articles published in this issue of SEAJME discuss excellent innovations covering the whole spectrum of medical education from undergraduate, postgraduate to continuous professional development (CPD). This digital transition will have a lasting and positive impact on medical education by increasing the breadth of teaching resources available (Tenzin et al 2020; Karunathilake et al, 2020).

Department of Medical Education, Faculty of Medicine, University of Colombo, Sri Lanka.

Corresponding author: Professor Indika Karunathilake Email: karunathilake@hotmail.com
The COVID-19 crisis has also provided several opportunities for professional development. For example, the increasing number of patients suffering from COVID-19 has led to redeployment of doctors from other specialties to help in management. While this is certainly challenging to those who may need to work in areas outside their comfort zone, it also provides training opportunities and revision of prior learning. The COVID-19 pandemic has been accompanied by an equally daunting infordemic, providing ample opportunities to practice evidence-based medicine. Medical professionals can use their training to critically analyse published materials against what is reported in the media and provide accurate health education (Yuen \& Xie, 2020).

As Medical Educationists we have the responsibility of identifying these opportunities and incorporating them to the medical education process.

\section{References}

Karunathilake, I., 2020. Beyond 2020: Moving Towards New Normal in Medical Education. South-East Asian Journal of Medical Education, 14(2), p.1.

Karunathilake, I.M., Dissanayake, V.H.W., Yasawardena, S., Abegunawardena, A., Raviraj, S., Wijesinghe, P.S., Anthony, A.A., Wijesinghe, R.A.N.K., Bowatte, S., Wickramaratne, N. and Pathirana, K.D., 2020. The new normal of medical educationchallenges and opportunities. South-East Asian Journal of Medical Education, 14(1)

Tenzin, K., Karunatilaka, I.M., Rimal, J., Wangdi, P., Gyamtsho, S., Tobgay, T., Dorji, G., Pradhan, D., Tshering, D., Supe, A. and Jayasinghe, S., (2020) The making of 21st century doctors of Bhutan; use of artificial intelligence, big data and values appropriate for the new normal in the 21st century. SouthEast Asian Journal of Medical Education, 14(1). 
Yuen J \& Xie F (2020) Medical education during the COVID-19 pandemic: perspectives from UK trainees, Postgraduate Medical Journal, 96, pp. 432-433. 